\title{
Pacific
}

Journal of

Mathematics

\section{FRACTAL ENTROPY OF NONAUTONOMOUS SYSTEMS}

Rui Kuang, Wen-Chiao Cheng and Bing Li 


\title{
FRACTAL ENTROPY OF NONAUTONOMOUS SYSTEMS
}

\author{
Rui KuANG, Wen-Chiao Cheng AND Bing Li
}

\begin{abstract}
We define formulas of entropy dimension for a nonautonomous dynamical system consisting of a sequence of continuous self-maps of a compact metric space. This study reveals analogues of basic propositions for entropy dimension, such as the power rule, product rule and commutativity, etc. These properties allow us to convert to an equality an inequality found by de Carvalho (1997) concerning the product rule for the autonomous dynamical system. We also prove a subadditivity rule of entropy dimension for one-dimensional dynamics based on our previous work.
\end{abstract}

\section{Introduction}

Entropies are important factors in the study of autonomous (i.e., deterministic) dynamical systems that are induced by iterations of a single transformation. The concept of topological entropy was originally introduced by Adler, Konheim and McAndrew [Adler et al. 1965] as an invariant of topological conjugacy and a numerical measure for the complexity of a dynamical system. Later on, Bowen [1971] and Dinaburg [1971] gave an equivalent definition when the space is metrizable. Other studies [Brucks and Bruin 2004; Katok and Hasselblatt 1995; Pollicott and Yuri 1998; Walters 1982] and the references therein discuss related definitions and properties. In the 1990s, various authors introduced several refinements of the notion of entropy, leading to significant findings in many different directions.

The commutativity formula for topological entropy (and measure theoretic entropy) was proved first in [Dana and Montrucchio 1986]. With the development of the study of nonautonomous dynamical systems, Kolyada and Snoha [1996] introduced and studied the notion of topological entropy for a sequence of continuous

This work was carried out while Cheng was partially supported by NSC grant 100-2115-M-034-001 and Li visited CMTP, National Central University. Li would like to thank the institute for their warm hospitality. Li was supported by the Fundamental Research Funds for the Central Universities (2011ZM0083, 2012ZB0018) and the Centre of Excellence in Analysis and Dynamics Research, Finland. Kuang was supported by NSFC $(10926038,10901080)$ and the Fundamental Research Funds for the Central Universities (2009ZM0325). Cheng is the corresponding author.

MSC2000: primary 37D35; secondary 37A35.

Keywords: entropy dimension, dynamical systems, nonautonomous dynamical systems, power rule, product rule, commutativity, subadditivity. 
self-maps of a compact metric space. Many properties for such dynamical systems were studied in [Cánovas 2011; Huang et al. 2008; Kolyada et al. 1999; 2004; Mouron 2007] and elsewhere. Particularly, the commutativity of the topological entropy was proved and announced in [Kolyada and Snoha 1996]. This kind of problem for nonautonomous dynamical systems has been studied for many years by several authors. A good discussion of these properties and applications appears in [Balibrea et al. 1999; Cánovas and Linero 2002; 2005; Hric 1999; 2000; Kolyada and Snoha 1996; Zhu et al. 2006].

Although systems with positive entropy are much more complicated than those with zero entropy, zero entropy systems have various complexities; see [de Carvalho 1997; Dou et al. 2011; Ferenczi and Park 2007; Huang et al. 2007; Misiurewicz 1981; Misiurewicz and Smítal 1988; Misiurewicz and Szlenk 1980]. These studies give some methods of classifying zero entropy dynamical systems. De Carvalho [1997] introduced a notion of entropy dimension to distinguish zero topological entropy systems and obtained some basic properties of entropy dimension. Cheng and $\mathrm{Li}[2010]$ presented some examples to show that every number in $(0,1)$ can be attained by the entropy dimensions of the dynamical systems and a dynamical system whose entropy dimension is one and topological entropy is zero. These findings answered the question asked in [de Carvalho 1997].

This paper analyzes a nonautonomous discrete dynamical system $\left(X, T_{1, \infty}\right)$ given by a compact metric space $X$ and a sequence $T_{1, \infty}=\left\{T_{i}\right\}_{i=1}^{\infty}$ of continuous self-maps of $X$. The trajectory of a fixed point $x$ is defined as the sequence $x, T_{1}(x), T_{2}\left(T_{1}(x)\right), \ldots$ Our goal is to study the properties of fractal entropy of nonautonomous dynamical systems. The paper is organized as follows. Section 2 defines and studies the entropy dimension $D\left(T_{1, \infty}\right)$ of a nonautonomous dynamical system given by a sequence $T_{1, \infty}=\left\{T_{i}\right\}_{i=1}^{\infty}$ of continuous maps of a compact metric space $X$ into itself. Section 3 investigates some formulas of entropy dimension for nonautonomous dynamical systems. These include the power rule, product rule and topological equisemiconjugacy. Applying these results shows that the commutativity of entropy dimension is also true for nonautonomous dynamical systems and the product rule holds for the autonomous dynamics, which was given just as an inequality in [de Carvalho 1997]. Section 4 focuses on continuous maps on the unit interval $[0,1]$. To show the subadditivity of entropy dimension, this paper uses the main result in [Cheng and $\mathrm{Li} 2010$ ] to consider two continuous commuting interval maps. Finally, we discuss the notion of the asymptotical entropy dimension.

\section{Equivalent definitions}

Topological entropy is one of the most fundamental dynamical invariants associated to a continuous map. It roughly measures the orbit structure complexity of the map. 
For nonautonomous dynamical systems, a sequence of continuous maps $\left\{T_{i}\right\}_{i=1}^{\infty}$ is considered. The $s$-topological entropy dimension of a nonautonomous dynamical system is introduced in this section. After that, we give different types of equivalent definitions.

Let $(X, d)$ be a compact metric space and $\left\{T_{i}\right\}_{i=1}^{\infty}$ be a sequence of continuous maps from $X$ to itself. Denote by $T_{1, \infty}$ the sequence $\left\{T_{i}\right\}_{i=1}^{\infty}$ and by $\left(X, T_{1, \infty}\right)$ the induced nonautonomous dynamical system.

For any $i \in \mathbb{N}$, let $T_{i}^{0}=\mathrm{Id}$, where Id is the identity map on $X$. Set

$$
T_{i}^{n}=T_{i+(n-1)} \circ \cdots \circ T_{i+1} \circ T_{i} \quad \text { and } \quad T_{i}^{-n}=T_{i}^{-1} \circ T_{i+1}^{-1} \circ \cdots \circ T_{i+(n-1)}^{-1} .
$$

For any open cover $\mathscr{A}$ of $X$, define

$$
T_{i}^{-n}(\mathscr{A})=\left\{T_{i}^{-n}(A): A \in \mathscr{A}\right\}
$$

and

$$
\begin{aligned}
\mathscr{A}_{i}^{n}\left(T_{1, \infty}\right) & =\bigvee_{j=0}^{n-1} T_{i}^{-j}(\mathscr{A}) \\
& =\left\{A_{i_{0}} \cap T_{i}^{-1}\left(A_{i_{1}}\right) \cap \cdots \cap T_{i}^{-(n-1)}\left(A_{i_{n-1}}\right): A_{i_{j}} \in \mathscr{A}, 1 \leq j \leq n-1\right\} .
\end{aligned}
$$

We write $\mathscr{A}_{1}^{n}$ for simplicity instead of $\mathscr{A}_{i}^{n}\left(T_{1, \infty}\right)$ if there is no confusion. Let $\mathcal{N}(\mathscr{A})$ be the minimal possible cardinality of a subcover chosen from $\mathscr{A}$.

Definition 2.1. Let $T_{i}: X \rightarrow X, i=1,2,3, \ldots$, be a sequence of continuous maps and $s \geq 0$ be a real number. The $s$-topological entropy of $T_{1, \infty}$ is defined as

$$
D\left(s, T_{1, \infty}\right)=\sup _{\mathscr{A}} D\left(s, T_{1, \infty}, \mathscr{A}\right),
$$

where $\mathscr{A}$ ranges over all open covers of $X$ and

$$
D\left(s, T_{1, \infty}, \mathscr{A}\right)=\limsup _{n \rightarrow \infty} \frac{1}{n^{s}} \log \mathcal{N}\left(\mathscr{A}_{1}^{n}\right) .
$$

When $T_{i}=T$ for all $i \in \mathbb{N}, D\left(s, T_{1, \infty}\right)$ is just the $s$-topological entropy of $T$ defined in [Cheng and Li 2010] (denoted by $D(s, T)$ ). Furthermore, if $s=1$ and $T_{i}=T$ for all $i \in \mathbb{N}$, it is trivial that $D\left(s, T_{i, \infty}\right)$ is just the topological entropy of $T$ (usually denoted by $h(T)$ ).

From Definition 2.1 it follows that the $s$-topological entropy $D\left(s, T_{1, \infty}\right)$ enjoys the following properties.

Proposition 2.2. (i) The map $s>0 \mapsto D\left(s, T_{1, \infty}\right)$ is nonnegative and decreasing with $s$.

(ii) There exists $s_{0} \in[0,+\infty]$ such that

$$
D\left(s, T_{1, \infty}\right)= \begin{cases}+\infty & \text { if } 0<s<s_{0}, \\ 0 & \text { if } s>s_{0} .\end{cases}
$$


Proposition 2.2(ii) indicates that the value of $D\left(s, T_{1, \infty}\right)$ jumps from infinity to 0 at both sides of some point $s_{0}$, which is similar to a fractal measure. Analogously to the fractal dimension, define the entropy dimension of $T_{1, \infty}$ as follows.

Definition 2.3. Let $\left(X, T_{1, \infty}\right)$ be a nonautonomous dynamical system. Define the entropy dimension of $T_{1, \infty}$ to be

$$
D\left(T_{1, \infty}\right)=\sup \left\{s>0: D\left(s, T_{1, \infty}\right)=\infty\right\}=\inf \left\{s>0: D\left(s, T_{1, \infty}\right)=0\right\} .
$$

When $T_{i}=T$ for all $i \in \mathbb{N}$, then $D\left(T_{1, \infty}\right)=D(T)$, where $D(T)$ is the entropy dimension of $T$ defined in [Cheng and Li 2010; Dou et al. 2011].

We now turn to definitions motivated by analogues of the topological entropy. Let $n \in \mathbb{N}$ and define a new (Bowen) metric $d_{n}$ on $X$ by

$$
d_{n}(x, y)=\max _{0 \leq i<n} d\left(T_{1}^{i}(x), T_{1}^{i}(y)\right),
$$

where $x, y \in X$.

Definition 2.4. A set $F \subset X$ is called an $(n, \varepsilon)$-spanning set of $X$ for $T_{1, \infty}$ if, for any $x \in X$, there exists $y \in F$ with $d_{n}(x, y) \leq \varepsilon$. A dual definition is as follows. A set $E \subset X$ is called an $(n, \varepsilon)$-separated set of $X$ for $T_{1, \infty}$ if $d_{n}(x, y)>\varepsilon$ for every pair of distinct point $x, y \in E, x \neq y$.

Define

$$
\begin{aligned}
& r\left(T_{1, \infty}, n, \varepsilon\right)=\min \left\{\# F: F \subset X \text { is an }(n, \varepsilon) \text {-spanning set for } T_{1, \infty}\right\}, \\
& s\left(T_{1, \infty}, n, \varepsilon\right)=\max \left\{\# E: E \subset X \text { is an }(n, \varepsilon) \text {-separated set for } T_{1, \infty}\right\},
\end{aligned}
$$

where $\# E$ is the number of elements in $E$. The following lemma describes the relationship among these two quantities and the number of covering sets.

Lemma 2.5. Let $T_{i}: X \rightarrow X$ be a sequence of continuous maps of a compact metric space $(X, d)$.

(i) For any open cover $\mathscr{A}$ of $X$ with Lebesgue number $\delta$,

$$
\mathcal{N}\left(\mathscr{A}_{1}^{n}\right) \leq r\left(T_{1, \infty}, n, \delta / 2\right) \leq s\left(T_{1, \infty}, n, \delta / 2\right) .
$$

(ii) For any $\varepsilon>0$ and open cover $\mathscr{A}$ with $\operatorname{diam}(\mathscr{A}):=\max \{\operatorname{diam}(A): A \in \mathscr{A}\} \leq \varepsilon$,

$$
r\left(T_{1, \infty}, n, \varepsilon\right) \leq s\left(T_{1, \infty}, n, \varepsilon\right) \leq \mathcal{N}\left(\mathscr{A}_{1}^{n}\right) .
$$

Proof. (i) Since any maximal $(n, \varepsilon)$-separated set of $X$ for $T_{1, \infty}$ is $(n, \varepsilon)$-spanning, the second inequality of (2-1) holds. Thus, it suffices to prove the first inequality. Let $F$ be a $(n, \delta / 2)$-spanning set for $X$ of cardinality $r\left(T_{1, \infty}, n, \delta / 2\right)$. Then

$$
X=\bigcup_{x \in F} \bigcap_{i=0}^{n-1} T_{1}^{-i} B\left(T_{1}^{i} x, \delta / 2\right) \text {. }
$$


Note that $B\left(T^{i} x, \delta / 2\right)$ is a subset of a member of $\mathscr{A}$ for any $0 \leq i \leq n-1$ and $x \in F$; thus,

$$
\mathcal{N}\left(\mathscr{A}_{1}^{n}\right) \leq r\left(T_{1, \infty}, n, \varepsilon\right) .
$$

(ii) The first inequality of (2-2) holds, as in (i). It suffices to prove the second inequality of (2-2). Let $E$ be an $(n, \varepsilon)$-separated set of cardinality $s\left(T_{1, \infty}, n, \varepsilon\right)$. Then no member of the cover $\mathscr{A}_{1}^{n}$ can contain two elements of $E$ since $\operatorname{diam}(\mathscr{A}) \leq \varepsilon$. This implies

$$
s\left(T_{1, \infty}, n, \varepsilon\right) \leq \mathcal{N}\left(\mathscr{A}_{1}^{n}\right) .
$$

Lemma 2.5 immediately implies the following property, which indicates that the $s$-topological entropy for $T_{1, \infty}$ can be equivalently defined by the spanning and separated sets.

Proposition 2.6. Let $T_{i}: X \rightarrow X, i=1,2,3, \ldots$, be a sequence of continuous maps and $s \geq 0$ a real number. Then

$$
D\left(s, T_{1, \infty}\right)=\lim _{\varepsilon \rightarrow 0} \limsup _{n \rightarrow \infty} \frac{1}{n^{s}} \log r\left(T_{1, \infty}, n, \varepsilon\right)=\lim _{\varepsilon \rightarrow 0} \limsup _{n \rightarrow \infty} \frac{1}{n^{s}} \log s\left(T_{1, \infty}, n, \varepsilon\right) .
$$

\section{Dynamical propositions}

The entropy dimension we defined for a nonautonomous dynamical system is a topological equiconjugacy invariant. Thus, we can consider those two entropy zero dynamical systems as being not the same or being not equivalent by different entropy dimension. The main idea of this section is quite similar to that of Kolyada and Snoha's approximations. The basic proposition of entropy dimension is the power rule. The inequality of the power rule can be shown as follows.

Lemma 3.1 [Kolyada and Snoha 1996]. Let $\mathscr{A}, \mathscr{B}$ be any two open covers of $X$. Then

(i) $\mathcal{N}(\mathscr{A} \vee \mathscr{B}) \leq \mathcal{N}(\mathscr{A}) \mathcal{N}(\mathscr{B})$;

(ii) $\mathcal{N}\left(T_{i}^{-n} \mathscr{A}\right) \leq \mathcal{N}(\mathscr{A})$;

(iii) $T^{-1}(\mathscr{A} \vee \mathscr{B})=T^{-1}(\mathscr{A}) \vee T^{-1}(\mathscr{B})$;

(iv) $\mathcal{N}(\mathscr{A}) \geq \mathcal{N}(\mathscr{B})$ when $\mathscr{A}$ is finer than $\mathscr{B}$ (denoted by $\mathscr{A} \succ \mathscr{B}$ ).

Proposition 3.2. Let $X$ be a compact topological space and $T_{1, \infty}$ a sequence of continuous maps from $X$ to itself. Then

$$
D\left(s, T_{1, \infty}^{m}\right) \leq m^{s} D\left(s, T_{1, \infty}\right)
$$

for any $s>0$ and $m \in \mathbb{N}$, where $T_{1, \infty}^{m}=\left\{T_{i m+1}^{(i+1) m}\right\}_{i=0}^{\infty}$. As a consequence,

$$
D\left(T_{1, \infty}^{m}\right) \leq D\left(T_{1, \infty}\right) \text {. }
$$


Proof. Let $\mathscr{A}$ be any open cover of $X$. For any $n \in \mathbb{N}$,

$$
\begin{aligned}
\mathscr{A} \vee T_{1}^{-1}(\mathscr{A}) \vee T_{1}^{-2}(\mathscr{A}) \vee \cdots \vee & T_{1}^{-(n m-1)}(\mathscr{A}) \\
& \succ \mathscr{A} \vee T_{1}^{-m}(\mathscr{A}) \vee T_{1}^{-2 m}(\mathscr{A}) \vee \cdots \vee T_{1}^{-(n-1) m}(\mathscr{A}),
\end{aligned}
$$

so by Lemma 3.1(iv),

$$
\mathcal{N}\left(\mathscr{A} \vee T_{1}^{-1}(\mathscr{A}) \vee \cdots \vee T_{1}^{-(n m-1)}(\mathscr{A})\right) \geq \mathcal{N}\left(\mathscr{A} \vee T_{1}^{-m}(\mathscr{A}) \vee \cdots \vee T_{1}^{-(n-1) m}(\mathscr{A})\right)
$$

Note that

$$
\begin{aligned}
\mathscr{A} \vee T_{1}^{-m}(\mathscr{A}) \vee T_{1}^{-2 m}(\mathscr{A}) \vee \cdots & \vee T_{1}^{-(n-1) m}(\mathscr{A}) \\
=\mathscr{A} \vee\left(T_{1}^{m}\right)^{-1}(\mathscr{A}) & \vee\left(T_{1}^{m}\right)^{-1} \circ\left(T_{m+1}^{m}\right)^{-1}(\mathscr{A}) \vee \cdots \\
& \vee\left(T_{1}^{m}\right)^{-1} \circ\left(T_{m+1}^{m}\right)^{-1} \circ \cdots \circ\left(T_{(n-2) m+1}^{m}\right)^{-1}(\mathscr{A}),
\end{aligned}
$$

and thus

$$
\begin{aligned}
\limsup _{n \rightarrow \infty} \frac{1}{(m n)^{s}} \log \mathcal{N}\left(\mathscr{A} \vee T_{1}^{-1}(\mathscr{A}) \vee T_{1}^{-2}(\mathscr{A}) \vee \cdots \vee T_{1}^{-(n m-1)}(\mathscr{A})\right) & \\
& \geq \frac{1}{m^{s}} D\left(s, T_{1, \infty}^{m}, \mathscr{A}\right) .
\end{aligned}
$$

Therefore,

$$
\begin{aligned}
D\left(s, T_{1, \infty}, \mathscr{A}\right) & =\limsup _{k \rightarrow \infty} \frac{1}{k^{s}} \log \mathcal{N}\left(\mathscr{A}_{1}^{k}\right) \geq \limsup _{n \rightarrow \infty} \frac{1}{(n m)^{s}} \log \mathcal{N}\left(\mathscr{A}_{1}^{n m}\right) \\
& =\limsup _{n \rightarrow \infty} \frac{1}{(n m)^{s}} \log \mathcal{N}\left(\mathscr{A} \vee T_{1}^{-1}(\mathscr{A}) \vee T_{1}^{-2}(\mathscr{A}) \vee \cdots \vee T_{1}^{-(n m-1)}(\mathscr{A})\right) \\
& \geq \frac{1}{m^{s}} D\left(s, T_{1, \infty}^{m}, \mathscr{A}\right) .
\end{aligned}
$$

Thus, $D\left(s, T_{1, \infty}^{m}\right) \leq m^{s} D\left(s, T_{1, \infty}\right)$.

For the entropy dimension, assume $t>D\left(T_{1, \infty}\right)$ is any real number. Then $D\left(t, T_{1, \infty}\right)=0$, which, combined with (3-1), implies $D\left(t, T_{1, \infty}^{m}\right)=0$, so $t \geq$ $D\left(T_{1, \infty}^{m}\right)$. Therefore, $D\left(T_{1, \infty}^{m}\right) \leq D\left(T_{1, \infty}\right)$ by the arbitrariness of $t$.

[Kolyada and Snoha 1996] gives an example showing that the inequality in (3-1) can be sharp when $s=1$. The following two propositions indicate that the inequality in (3-1) can be an equality under some conditions.

Proposition 3.3 (power rule). Let $X$ be a compact topological space and $T_{1, \infty}$ be a sequence of continuous maps from $X$ to itself. If $T_{1, \infty}$ is periodic with period $m \in \mathbb{N}$, that is, $T_{i m+j}=T_{j}$ for any $1 \leq j \leq m$ and $i \geq 0$, then

$$
D\left(s, T_{1, \infty}^{m}\right)=m^{s} D\left(s, T_{1, \infty}\right)
$$

for any $s>0$. As a consequence, $D\left(T_{1, \infty}^{m}\right)=D\left(T_{1, \infty}\right)$. 
Proof. Assume $m \geq 2$ since the case $m=1$ is trivial. From Proposition 3.2, it is only necessary to prove $D\left(s, T_{1, \infty}^{m}\right) \geq m^{s} D\left(s, T_{1, \infty}\right)$.

Let $\mathscr{A}$ be any open cover of $X$ and $k=n m+r$, where $n \geq 1$ and $1 \leq r \leq m$. Combining $T_{1, \infty}=\left\{T_{1}, T_{2}, \ldots, T_{m}, T_{1}, T_{2}, \ldots, T_{m}, \ldots\right\}$ and $T_{1, \infty}^{m}=\left\{T_{1}^{m}, T_{1}^{m}, \ldots\right\}$ with Lemma 3.1(iii),

$$
\begin{aligned}
T_{1}^{-i m}(\mathscr{A}) \vee T_{1}^{-(i m+1)}(\mathscr{A}) \vee \cdots \vee & T_{1}^{-((i+1) m-1)}(\mathscr{A}) \\
& =T_{1}^{-i m}\left(\mathscr{A} \vee T_{i m+1}^{-1}(\mathscr{A}) \vee \cdots \vee T_{i m+1}^{-(m-1)}(\mathscr{A})\right) \\
& =T_{1}^{-i m}\left(\mathscr{A} \vee T_{1}^{-1}(\mathscr{A}) \vee \cdots \vee T_{1}^{-(m-1)}(\mathscr{A})\right) \\
& =\left(T_{1}^{i m}\right)^{-1}\left(\mathscr{A} \vee T_{1}^{-1}(\mathscr{A}) \vee \cdots \vee T_{1}^{-(m-1)}(\mathscr{A})\right)
\end{aligned}
$$

for $i=0,1,2, \ldots$ Therefore, $\mathscr{A}_{1}^{k}\left(T_{1, \infty}\right)$ can be written as

$$
\begin{gathered}
\left(\mathscr{A} \vee T_{1}^{-1}(\mathscr{A}) \vee \cdots \vee T_{1}^{-(m-1)}(\mathscr{A})\right) \vee\left(T_{1}^{-m}(\mathscr{A}) \vee T_{1}^{-(m+1)}(\mathscr{A}) \vee \cdots \vee T_{1}^{-(2 m-1)}(\mathscr{A})\right) \\
\vee \cdots \vee\left(T_{1}^{-(n-1) m} \mathscr{A} \vee T_{1}^{-((n-1) m+1)}(\mathscr{A}) \vee \cdots \vee T_{1}^{-(n m-1)}(\mathscr{A})\right) \\
\vee\left(T_{1}^{-n m} \mathscr{A} \vee T_{1}^{-(n m+1)}(\mathscr{A}) \vee \cdots \vee T_{1}^{-(n m+r-1)}(\mathscr{A})\right) \\
=\left(\mathscr{A} \vee T_{1}^{-1}(\mathscr{A}) \vee \cdots \vee T_{1}^{-(m-1)}(\mathscr{A})\right) \vee\left(T_{1}^{m}\right)^{-1}\left(\mathscr{A} \vee T_{1}^{-1}(\mathscr{A}) \vee \cdots \vee T_{1}^{-(m-1)}(\mathscr{A})\right) \\
\vee \cdots \vee\left(T_{1}^{(n-1) m}\right)^{-1}\left(\mathscr{A} \vee T_{1}^{-1}(\mathscr{A}) \vee \cdots \vee T_{1}^{-(m-1)}(\mathscr{A})\right) \\
\vee\left(T_{1}^{n m}\right)^{-1}\left(\mathscr{A} \vee T_{1}^{-1}(\mathscr{A}) \vee \cdots \vee T_{1}^{-(r-1)}(\mathscr{A})\right) \\
=\mathscr{A}_{1}^{m} \vee\left(T_{1}^{m}\right)^{-1}\left(\mathscr{A}_{1}^{m}\right) \vee \cdots \vee\left(T_{1}^{(n-1) m}\right)^{-1}\left(\mathscr{A}_{1}^{m}\right) \vee\left(T_{1}^{n m}\right)^{-1}\left(\mathscr{A}_{1}^{r}\right) \\
=\left(\mathscr{A}_{1}^{m}\left(T_{1, \infty}\right)\right)_{1}^{n}\left(T_{1, \infty}^{m}\right) \vee\left(T_{1}^{n m}\right)^{-1}\left(\mathscr{A}_{1}^{r}\left(T_{1, \infty}\right)\right) .
\end{gathered}
$$

Combining parts (i) and (iii) of Lemma 3.1, we obtain

$$
\begin{aligned}
\mathcal{N}\left(\mathscr{A}_{1}^{n m+r}\left(T_{1, \infty}\right)\right) & =\mathcal{N}\left(\left(\mathscr{A}_{1}^{m}\left(T_{1, \infty}\right)\right)_{1}^{n}\left(T_{1, \infty}^{m}\right) \vee\left(T_{1}^{n m}\right)^{-1}\left(\mathscr{A}_{1}^{r}\left(T_{1, \infty}\right)\right)\right) \\
& \leq \mathcal{N}\left(\left(\mathscr{A}_{1}^{m}\left(T_{1, \infty}\right)\right)_{1}^{n}\left(T_{1, \infty}^{m}\right)\right) \mathcal{N}\left(\mathscr{A}_{1}^{r}\left(T_{1, \infty}\right)\right) .
\end{aligned}
$$

Thus,

$$
\begin{aligned}
D\left(s, T_{1, \infty}^{m}, \mathscr{A}_{1}^{m}\left(T_{1, \infty}\right)\right) & =\limsup _{n \rightarrow \infty} n^{-s} \log \mathcal{N}\left(\left(\mathscr{A}_{1}^{m}\left(T_{1, \infty}\right)\right)_{1}^{n}\left(T_{1, \infty}^{m}\right)\right) \\
& \geq \limsup _{n \rightarrow \infty} n^{-s}\left(\log \mathcal{N}\left(\mathscr{A}_{1}^{n m+r}\left(T_{1, \infty}\right)\right)-\log \mathcal{N}\left(\mathscr{A}_{1}^{r}\left(T_{1, \infty}\right)\right)\right) \\
& =\limsup _{n \rightarrow \infty} n^{-s} \log \mathcal{N}\left(\mathscr{A}_{1}^{n m+r}\left(T_{1, \infty}\right)\right) \\
& =m^{s} \limsup _{n \rightarrow \infty}(n m+r)^{-s} \log \mathcal{N}\left(\mathscr{A}_{1}^{n m+r}\left(T_{1, \infty}\right)\right) \\
& =m^{s} \limsup _{k \rightarrow \infty} k^{-s} \log \mathcal{N}\left(\mathscr{A}_{1}^{k}\left(T_{1, \infty}\right)\right)=m^{s} D\left(s, T_{1, \infty}, \mathscr{A}\right),
\end{aligned}
$$

which implies that $D\left(s, T_{1, \infty}^{m}\right) \geq m^{s} D\left(s, T_{1, \infty}\right)$ by the arbitrariness of $\mathscr{A}$. 
Applying Proposition 3.3 to the case of one map as a sequence leads to the following, which solves a problem in [de Carvalho 1997], where the author gave an inequality.

Corollary 3.4. Let $(X, T)$ be a topological dynamical system. Then

$$
D\left(s, T^{m}\right)=m^{s} D(s, T)
$$

for any $s>0$ and $m \in \mathbb{N}$. In particular, $D\left(T^{m}\right)=D(T)$.

Now let us consider the sequence of equicontinuous maps from $X$ to itself; that is, $T_{1, \infty}=\left\{T_{i}\right\}_{i=1}^{\infty}$ is equicontinuous on $X$. More precisely, for any $x \in X$ and $\varepsilon>0$, there exists $\delta>0$ such that $d\left(T_{i} x, T_{i} y\right)<\varepsilon$ for all $i=1,2, \ldots$ whenever $d(x, y)<\delta$. We know that $\delta$ can be independent of the choice of $x$ when $X$ is compact.

Proposition 3.5 (power rule). Let $(X, d)$ be a compact metric space and $T_{1, \infty}$ be a sequence of equicontinuous maps from $X$ to itself. Then

$$
D\left(s, T_{1, \infty}^{m}\right)=m^{s} D\left(s, T_{1, \infty}\right)
$$

for any $s>0$.

Proof. By Proposition 3.2, it suffices to prove $D\left(s, T_{1, \infty}^{m}\right) \geq m^{s} D\left(s, T_{1, \infty}\right)$ for $m \geq 2$. For any $\varepsilon>0$, let

$$
\delta(\varepsilon)=\varepsilon+\sup _{i \geq 1} \max _{k=1, \ldots, m-1} \sup _{x, y \in X}\left\{d\left(T_{i}^{k}(x), T_{i}^{k}(y)\right): d(x, y) \leq \varepsilon\right\} .
$$

Since $X$ is compact and $T_{1, \infty}$ is equicontinuous, we have:

(i) if $\varepsilon \rightarrow 0$, then $\delta(\varepsilon) \rightarrow 0$;

(ii) if $d(x, y) \leq \varepsilon$, then $d\left(T_{i}^{k}(x), T_{i}^{k}(y)\right) \leq \delta(\varepsilon)$ for any $i \geq 1$ and $k=1,2, \ldots, m-1$.

Let $E$ be any $(n m, \delta(\varepsilon))$-separated set for $T_{1, \infty}$. Then, $E$ is an $(n, \varepsilon)$-separated set for $T_{1, \infty}^{m}$ and $s_{n m}\left(T_{1, \infty}, \delta(\varepsilon)\right) \leq s_{n}\left(T_{1, \infty}^{m}, \varepsilon\right)$.

Therefore, writing $k=n m+r$ with $1 \leq r \leq m$, we have the following calculation:

$$
\begin{aligned}
D\left(s, T_{1, \infty}^{m}\right) & =\lim _{\varepsilon \rightarrow 0} \limsup _{n \rightarrow \infty} \frac{1}{n^{s}} \log s_{n}\left(T_{1, \infty}^{m}, \varepsilon\right) \\
& \geq \lim _{\varepsilon \rightarrow 0} \limsup _{n \rightarrow \infty} \frac{1}{n^{s}} \log s_{(n-1) m+r}\left(T_{1, \infty}, \delta(\varepsilon)\right) \\
& =m^{s} \lim _{\varepsilon \rightarrow 0} \limsup _{n \rightarrow \infty} \frac{1}{((n-1) m+r)^{s}} \log s_{(n-1) m+r}\left(T_{1, \infty}, \delta(\varepsilon)\right) \\
& \geq m^{s} \lim _{\delta(\varepsilon) \rightarrow 0} \limsup _{k \rightarrow \infty} \frac{1}{k^{s}} \log s_{k}\left(T_{1, \infty}, \delta(\varepsilon)\right) \\
& =m^{s} D\left(s, T_{1, \infty}\right) .
\end{aligned}
$$


Proposition 3.6 (monotonicity). Let $X$ be a compact topological space and $T_{1, \infty}$ a sequence of continuous maps from $X$ to itself. Then

$$
D\left(s, T_{i, \infty}\right) \leq D\left(s, T_{j, \infty}\right)
$$

for any $s>0$ and $1 \leq i \leq j \leq+\infty$.

Proof. Let $\mathscr{A}$ be any open cover of $X$. Lemma 3.1(i) implies

$$
\mathcal{N}\left(\mathscr{A}_{i}^{n}\right)=\mathcal{N}\left(\bigvee_{j=0}^{n-1} T_{i}^{-j}(\mathscr{A})\right)=\mathcal{N}\left(\mathscr{A} \vee \bigvee_{j=1}^{n-1} T_{i}^{-j}(\mathscr{A})\right) \leq \mathcal{N}(\mathscr{A}) \mathcal{N}\left(\bigvee_{j=1}^{n-1} T_{i}^{-j}(\mathscr{A})\right)
$$

Lemma 3.1(ii) shows that

$$
\mathcal{N}\left(\bigvee_{j=1}^{n-1} T_{i}^{-j}(\mathscr{A})\right)=\mathcal{N}\left(T_{i}^{-1}\left(\bigvee_{j=0}^{n-2} T_{i+1}^{-j}(\mathscr{A})\right)\right) \leq \mathcal{N}\left(\bigvee_{j=0}^{n-2} T_{i+1}^{-j}(\mathscr{A})\right)=\mathcal{N}\left(\mathscr{A}_{i+1}^{n-2}\right)
$$

Combining (3-3) and (3-4) leads to

$$
\mathcal{N}\left(\mathscr{A}_{i}^{n}\right) \leq \mathcal{N}(\mathscr{A}) \mathcal{N}\left(\mathscr{A}_{i+1}^{n-2}\right) .
$$

Therefore,

$$
D\left(s, T_{i, \infty}, \mathscr{A}\right)=\limsup _{n \rightarrow \infty} \frac{1}{n^{s}} \log \mathcal{N}\left(\mathscr{A}_{i}^{n}\right) \leq \limsup _{n \rightarrow \infty} \frac{1}{n^{s}} \log \left(\mathcal{N}(\mathscr{A}) \mathcal{N}\left(\mathscr{A}_{i+1}^{n-2}\right)\right) .
$$

Thus,

$$
D\left(s, T_{i, \infty}, \mathscr{A}\right) \leq \limsup _{n \rightarrow \infty} \frac{1}{(n-2)^{s}} \log \mathcal{N}\left(\mathscr{A}_{i+1}^{n-2}\right)=D\left(s, T_{i+1, \infty}, \mathscr{A}\right),
$$

and $D\left(s, T_{i, \infty}\right) \leq D\left(s, T_{i+1, \infty}\right)$ by the arbitrariness of $\mathscr{A}$. Hence, (3-2) holds.

Applying the monotonicity shows that the $s$-topological entropy for the composition of two maps does not depend on the order, as the following theorem indicates.

Theorem 3.7 (commutativity). Let $X$ be a compact topological space and let $T, S$ be two continuous maps from $X$ to itself. Then

$$
D(s, T \circ S)=D(s, S \circ T)
$$

for any $s>0$.

Proof. From Proposition 3.6, we obtain

$$
D(s,\{S, T, S, T, \ldots\}) \leq D(s,\{T, S, T, S, \ldots\}) \leq D(s,\{S, T, S, T \ldots\}),
$$

which implies

$$
D(s,\{S, T, S, T, \ldots\})=D(s,\{T, S, T, S, \ldots\}) .
$$


By Proposition 3.3,

$$
\begin{aligned}
D(s, T \circ S) & =D(s,\{T \circ S, T \circ S, \ldots\})=2^{s} D(s,\{S, T, S, T, \ldots\}) \\
& =2^{s} D(s,\{T, S, T, S, \ldots\})=D(s,\{S \circ T, S \circ T, \ldots\})=D(s, S \circ T) .
\end{aligned}
$$

Corollary 3.8. Let $X$ be a compact topological space and $T_{i}(i=1,2, \ldots, n)$ be the continuous self-maps on $X$. Then, for any $1<i \leq n$ and $s>0$,

$$
D\left(s, T_{n} \circ \cdots \circ T_{2} \circ T_{1}\right)=D\left(s, T_{i-1} \circ \cdots \circ T_{2} \circ T_{1} \circ T_{n} \circ \cdots \circ T_{i}\right) .
$$

Proof. By Theorem 3.7,

$$
\begin{aligned}
D\left(s, T_{n} \circ \cdots \circ T_{i} \circ T_{i-1} \circ \cdots \circ T_{2} \circ T_{1}\right) & =D\left(s,\left(T_{n} \circ \cdots \circ T_{i}\right) \circ\left(T_{i-1} \circ \cdots \circ T_{2} \circ T_{1}\right)\right) \\
& =D\left(s,\left(T_{i-1} \circ \cdots \circ T_{2} \circ T_{1}\right) \circ\left(T_{n} \circ \cdots \circ T_{i}\right)\right) \\
& =D\left(s, T_{i-1} \circ \cdots \circ T_{2} \circ T_{1} \circ T_{n} \circ \cdots \circ T_{i}\right) .
\end{aligned}
$$

The following corollary was given in [Cheng and Li 2010]; however, this paper provides a quick proof from the commutativity (Theorem 3.7).

Corollary 3.9. Let $X$ be a compact topological spaces and $T_{1}, T_{2}$ be two continuous maps on $X$. If $\left(X, T_{1}\right)$ is conjugate to $\left(Y, T_{2}\right)$, then $D\left(s, T_{1}\right)=D\left(s, T_{2}\right)$ for any $s>0$.

Proof. Let $\phi$ be a conjugacy between $T_{1}$ and $T_{2}$. Since $T_{2}=\phi \circ T_{1} \circ \phi^{-1}$, Theorem 3.7 shows that

$$
D\left(s, T_{2}\right)=D\left(s,\left(\phi \circ T_{1}\right) \circ \phi^{-1}\right)=D\left(s, T_{1}\right) .
$$

As Corollary 3.9 shows, the $s$-topological entropy $D(s, T)$ for an autonomous dynamical system is a conjugate invariant quantity. For the nonautonomous case, the definition of conjugacy must be adapted to the following.

Definition 3.10. Let $\left(X,\left\{T_{i}\right\}_{i=1}^{\infty}\right)$ and $\left(Y,\left\{S_{i}\right\}_{i=1}^{\infty}\right)$ be two nonautonomous dynamical systems. Denote by $\pi_{1, \infty}=\left\{\pi_{i}\right\}_{i=1}^{\infty}$ a sequence of equicontinuous surjective maps from $X$ to $Y$. If

$$
\pi_{i+1} \circ T_{i}=S_{i} \circ \pi_{i}
$$

for every $i \geq 1$, we say that $\pi_{1, \infty}$ is a topological equisemiconjugacy between $T_{1, \infty}$ and $S_{1, \infty}$, and the dynamical system $\left(X, T_{1, \infty}\right)$ is topologically equisemiconjugate with $\left(Y, S_{1, \infty}\right)$. Furthermore, if $\pi_{1, \infty}$ is an equicontinuous sequence of homeomorphisms such that the sequence $\pi_{1, \infty}^{-1}=\left\{\pi_{i}^{-1}\right\}_{i=1}^{\infty}$ of inverse homeomorphisms is also equicontinuous, we say that $\pi_{1, \infty}$ is a topological equiconjugacy between $T_{1, \infty}$ and $S_{1, \infty}$, and the dynamical system $\left(X, T_{1, \infty}\right)$ is topologically equiconjugate with $\left(Y, S_{1, \infty}\right)$. 
Theorem 3.11. Let $(X, d)$ and $(Y, \rho)$ be compact metric spaces and $T_{1, \infty}$ and $S_{1, \infty}$ be the sequences of continuous maps from $X$ and $Y$ into themselves, respectively. If the system $\left(X, T_{1, \infty}\right)$ is equisemiconjugate with $\left(Y, S_{1, \infty}\right)$, then

$$
D\left(s, S_{1, \infty}\right) \leq D\left(s, T_{1, \infty}\right)
$$

for any $s>0$.

Proof. Let $\pi_{1, \infty}$ be the equisemiconjugacy between $X$ and $Y$. For any given $\varepsilon>0$, noting that $\pi_{1, \infty}$ is a sequence of equicontinuous maps from $X$ onto $Y$ and $X$ is compact, there exists $\delta(\varepsilon)>0$ such that if $\rho\left(\pi_{i}(x), \pi_{i}(y)\right)>\varepsilon$ for some $i \geq 1$, then $d(x, y)>\delta(\varepsilon)$. Let $E \subset Y$ be an $(n, \varepsilon)$-separated set for $S_{1, \infty}$ with maximal cardinality $s\left(S_{1, \infty}, n, \varepsilon\right)$. Choose one point from each fiber $\pi_{1}^{-1}(y), y \in E$ and denote by $E_{X}$ the set of such points. Then $E_{X} \subset X$ is an $(n, \delta(\varepsilon))$-separated set for $T_{1, \infty}$. Therefore, $s\left(T_{1, \infty}, n, \delta(\varepsilon)\right) \geq s\left(S_{1, \infty}, n, \varepsilon\right)$, which implies (3-5).

Apply Theorem 3.11, the following statement holds.

Corollary 3.12. Let $(X, d)$ and $(Y, \rho)$ be compact metric spaces and $T_{1, \infty}$ and $S_{1, \infty}$ be the sequences of continuous maps from $X$ and $Y$ into themselves, respectively. If the system $\left(X, T_{1, \infty}\right)$ is equiconjugate with $\left(Y, S_{1, \infty}\right)$, then

$$
D\left(s, S_{1, \infty}\right)=D\left(s, T_{1, \infty}\right)
$$

for any $s>0$. As a result, $D\left(S_{1, \infty}\right)=D\left(T_{1, \infty}\right)$.

Theorem 3.13 (product rule). Let $(X, d)$ and $(Y, \rho)$ be compact metric spaces. Let $\left\{T_{i}\right\}_{i=1}^{\infty}$ and $\left\{S_{i}\right\}_{i=1}^{\infty}$ be two sequences of continuous maps on $X$ and $Y$, respectively. Define a metric $d^{*}$ on $X \times Y$ by $d^{*}\left(\left(x_{1}, y_{1}\right),\left(x_{2}, y_{2}\right)\right)=\max \left\{d\left(x_{1}, x_{2}\right), \rho\left(y_{1}, y_{2}\right)\right\}$ and a sequence of transformations on $X \times Y$ by $\left(T_{i} \times S_{i}\right)(x, y)=\left(T_{i} x, S_{i} y\right)$. Then

$$
D\left(s, T_{1, \infty} \times S_{1, \infty}\right) \leq D\left(s, T_{1, \infty}\right)+D\left(s, S_{1, \infty}\right)
$$

for any $s>0$, where $T_{1, \infty} \times S_{1, \infty}=\left\{T_{i} \times S_{i}\right\}_{i=1}^{\infty}$.

Proof. We know that balls in the $n$-Bowen metric $d_{n}^{*}$ are products of balls on $X$ and $Y$ since balls in the product metric $d^{*}$ are products of balls on $X$ and $Y$. Therefore,

$$
r\left(T_{1, \infty} \times S_{1, \infty}, n, \varepsilon\right) \leq r\left(T_{1, \infty}, n, \varepsilon\right) r\left(S_{1, \infty}, n, \varepsilon\right) .
$$

Thus $D\left(s, T_{1, \infty} \times S_{1, \infty}\right) \leq D\left(s, T_{1, \infty}\right)+D\left(s, S_{1, \infty}\right)$.

\section{Subadditivity}

For $S, T$ two continuous functions from the compact metric space $X$ to itself, some additional conditions are necessary to obtain some interesting results. It is natural to assume that $S$ and $T$ commute, that is, $S \circ T=T \circ S$. For instance, in [Hu 1993], the subadditivity of topological entropy $h(S \circ T) \leq h(S)+h(T)$ was proved 
for diffeomorphisms on $C^{\infty}$ compact Riemannian manifolds. This section also investigates the subadditivity for entropy dimension in one-dimensional dynamics. For convenience, the following two definitions use the same concept and notation adopted in [Cheng and Li 2010].

Definition 4.1. An interval map $T:[0,1] \rightarrow[0,1]$ is called piecewise monotone continuous if there exist points $0=a_{0}<a_{1}<\cdots<a_{N}=1$ such that $\left.T\right|_{\left(a_{i-1}, a_{i}\right)}$ is continuous and monotone.

Definition 4.2. Let $T$ be a piecewise monotone continuous map. If $J$ is a maximal interval on which $\left.T\right|_{J}$ is continuous and monotone, then $T: J \rightarrow T(J)$ is called a branch or lap of $T$. The number of laps of $T$ is denoted by $l(T)$.

Rothschild [1971] and Misiurewicz and Szlenk [1980] independently obtained the topological entropy formula for a piecewise monotone map (see [Brucks and Bruin 2004; Pollicott and Yuri 1998]). The following theorem gives a generalized $s$-topological entropy formula.

Theorem 4.3 [Cheng and Li 2010]. Let $T:[0,1] \rightarrow[0,1]$ be a piecewise monotone continuous map and $s>0$ a real number. Then

$$
D(s, T)=\limsup _{n \rightarrow \infty} \frac{\log l\left(T^{n}\right)}{n^{s}} .
$$

Theorem 4.4 (subadditivity). Let $T, S$ be piecewise monotone continuous maps such that $T \circ S=S \circ T$ and let $s>0$ be a real number. Then

$$
D(s, S \circ T) \leq D(s, S)+D(s, T) .
$$

Hence, we have the inequality

$$
D(S \circ T) \leq \max \{D(S), D(T)\} .
$$

Proof. Since $S \circ T=T \circ S$, it is trivial that $S^{p} \circ T^{q}=T^{q} \circ S^{p}$ for all $p, q \in \mathbb{N}$.

The number of intervals of monotonicity of $S^{n} \circ T^{n}$ is smaller than or equal to $l\left(T^{n}\right) l\left(S^{n}\right)$. Thus, we obtain that $l\left((S \circ T)^{n}\right) \leq l\left(S^{n}\right) l\left(T^{n}\right)$. The previous theorem gives that

$$
\begin{aligned}
D(s, S \circ T) & =\limsup _{n \rightarrow \infty} \frac{\log l\left((S \circ T)^{n}\right)}{n^{s}} \leq \limsup _{n \rightarrow \infty} \frac{\log l\left(S^{n}\right) l\left(T^{n}\right)}{n^{s}} \\
& \leq \limsup _{n \rightarrow \infty} \frac{\log l\left(S^{n}\right)}{n^{s}}+\limsup _{n \rightarrow \infty} \frac{\log l\left(T^{n}\right)}{n^{s}}=D(s, S)+D(s, T) .
\end{aligned}
$$

For any $t>\max \{D(S), D(T)\}$, it is clear that $D(t, S)=D(t, T)=0$ by the definition of entropy dimension. Thus, $D(t, S \circ T)=0$, which implies $D(S \circ T) \leq t$. It follows that $D(S \circ T) \leq \max \{D(S), D(T)\}$ by the arbitrariness of $t$. 
Corollary 4.5. Let $T, S$ be piecewise monotone continuous maps such that $T \circ S=$ $S \circ T$. If $D(S)=D(T)=0$, then $D(S \circ T)=0$.

Note that in general from $D(S)>0$ or $D(T)>0$, it may not possible to deduce that $D(S \circ T)>0$. To find a result in this setting, calculate the left shift $S$ and right shift $T$ on the symbolic space $\{1,2\}^{\mathbb{Z}}$. Then $S \circ T$ is the identity map. It is trivial that $D(S)=1$ and $D(T)=1$. However, $D(S \circ T)=0$. This example also indicates that the inequality in (4-2) can be sharp. On the other hand, it is easy to see that the inequality can be an equality. For example, if $S$ is the identity map, then $D(S)=0$, and $D(S \circ T)=D(T)=\max \{D(S), D(T)\}$. Some related properties of topological entropy of composition, $S \circ T$, can be found in [Goodwyn 1972; Raith 2004].

Consider a sequence $T_{1, \infty}=\left\{T_{i}\right\}_{i=1}^{\infty}$ of continuous functions from a compact metric space $X$ to itself. Proposition 3.6 shows a kind of monotonicity of $\left\{D\left(s, T_{i, \infty}\right)\right\}$ on $i \in \mathbb{N}$. Here, we can introduce the notion of the asymptotical entropy dimension of the considered system as the limit of entropy dimension in

$$
D^{*}\left(T_{\infty}\right)=\lim _{i \rightarrow \infty} D\left(T_{i, \infty}\right),
$$

where $T_{i, \infty}$ is the tail $T_{i}, T_{i+1}, \ldots$ of the sequence $T_{1, \infty}$.

Theorem 4.6. Let $T_{1, \infty}=\left\{T_{i}\right\}_{i=1}^{\infty}$ be a sequence of monotone continuous functions from $X$ to itself, where $X$ is the unit interval $[0,1]$ or unit circle $S^{1}$. Then the entropy dimension is $D\left(T_{1, \infty}\right)=0$. Consequently, $D^{*}\left(T_{\infty}\right)=0$.

Proof. Consider the unit interval case first. Assume that $E=\left\{x_{1}, x_{2}, \ldots, x_{k}\right\}$ is a subset of $[0,1]$ with $x_{1} \leq x_{2} \leq \cdots \leq x_{k}$. Since the functions $T_{1}, T_{2}, T_{3}, \ldots$ are monotone, for every $j=0,1,2,3, \ldots$, we obtain either

$$
T_{1}^{j}\left(x_{1}\right) \leq T_{1}^{j}\left(x_{2}\right) \leq T_{1}^{j}\left(x_{3}\right) \leq \cdots \leq T_{1}^{j}\left(x_{k}\right)
$$

or

$$
T_{1}^{j}\left(x_{1}\right) \geq T_{1}^{j}\left(x_{2}\right) \geq T_{1}^{j}\left(x_{3}\right) \geq \cdots \geq T_{1}^{j}\left(x_{k}\right) .
$$

This implies that the set $E$ is an $(n, \epsilon)$-separated set if and only if for every $i=1,2, \ldots, k-1$, the set $\left\{x_{i}, x_{i+1}\right\}$ is $(n, \epsilon)$-separated. Denote the integer part of a number $z$ by $[z]$. Since the length of the unit interval $[0,1]$ is 1 , at most $\left[\frac{1}{\epsilon}\right]$ of the distances $\left|T_{1}^{j}\left(x_{1}\right)-T_{1}^{j}\left(x_{2}\right)\right|,\left|T_{1}^{j}\left(x_{2}\right)-T_{1}^{j}\left(x_{3}\right)\right|, \ldots,\left|T_{1}^{j}\left(x_{k-1}\right)-T_{1}^{j}\left(x_{k}\right)\right|$ are longer than $\epsilon$. Therefore, at most $n\left[\frac{1}{\epsilon}\right]$ sets of the form $\left\{x_{i}, x_{i+1}\right\}, i=1,2, \ldots, k-1$ are $(n, \epsilon)$-separated. Thus, if $E$ is $(n, \epsilon)$-separated, then $k-1 \leq n\left[\frac{1}{\epsilon}\right]$. By definition, $D\left(s, T_{1, \infty}\right)=0$ for any $s>0$, which implies $D\left(T_{1, \infty}\right)=0$. Similarly, $D\left(T_{j, \infty}\right)=0$ for any $j>1$. Thus, $D^{*}\left(T_{\infty}\right)=0$

Next, consider the case $X=S^{1}$. The proof is similar to that of the unit interval case when the order of the points on $S^{1}$ is the angle of points on $S^{1}$. Therefore, $D^{*}\left(T_{\infty}\right)=0$ is also obtained in this case. 


\section{References}

[Adler et al. 1965] R. L. Adler, A. G. Konheim, and M. H. McAndrew, "Topological entropy", Trans. Amer. Math. Soc. 114 (1965), 309-319. MR 30 \#5291 Zbl 0127.13102

[Balibrea et al. 1999] F. Balibrea, V. Jiménez López, and J. S. Cánovas Peña, "Some results on entropy and sequence entropy”, Internat. J. Bifur. Chaos Appl. Sci. Engrg. 9:9 (1999), 1731-1742. MR 2000j:37008 Zbl 1089.37501

[Bowen 1971] R. Bowen, "Entropy for group endomorphisms and homogeneous spaces", Trans. Amer. Math. Soc. 153 (1971), 401-414. Erratum in 181 (1971), 509-510. MR 43 \#469 Zbl 0212.29201

[Brucks and Bruin 2004] K. M. Brucks and H. Bruin, Topics from one-dimensional dynamics, London Mathematical Society Student Texts 62, Cambridge University Press, 2004. MR 2005j:37048 Zbl 1074.37022

[Cánovas 2011] J. S. Cánovas, "Li-Yorke chaos in a class of nonautonomous discrete systems", J. Difference Equ. Appl. 17:4 (2011), 479-486. MR 2012c:37032 Zbl 1221.37080

[Cánovas and Linero 2002] J. S. Cánovas and A. Linero, "On topological entropy of commuting interval maps”, Nonlinear Anal. 51:7 (2002), 1159-1165. MR 2003g:37071 Zbl 1016.37008

[Cánovas and Linero 2005] J. S. Cánovas and A. Linero, "On the dynamics of composition of commuting interval maps", J. Math. Anal. Appl. 305:1 (2005), 296-303. MR 2005m:37078 Zbl 1063.37031

[de Carvalho 1997] M. de Carvalho, "Entropy dimension of dynamical systems", Portugal. Math. 54:1 (1997), 19-40. MR 98d:54075 Zbl 0965.37021

[Cheng and Li 2010] W.-C. Cheng and B. Li, "Zero entropy systems", J. Stat. Phys. 140:5 (2010), 1006-1021. MR 2012f:37011 Zbl 1198.37007

[Dana and Montrucchio 1986] R.-A. Dana and L. Montrucchio, "Dynamic complexity in duopoly games", J. Econom. Theory 40:1 (1986), 40-56. MR 87k:90045 Zbl 0617.90104

[Dinaburg 1971] E. I. Dinaburg, "On the relations among various entropy characteristics of dynamical systems”, Izv. Akad. Nauk SSSR Ser. Mat. 35 (1971), 324-366. In Russian; translated in Math. USSR Izv. 5:2 (1971), 337-378. MR 44 \#3307 Zbl 0248.58007

[Dou et al. 2011] D. Dou, W. Huang, and K. K. Park, "Entropy dimension of topological dynamical systems", Trans. Amer. Math. Soc. 363:2 (2011), 659-680. MR 2012a:37030 Zbl 1217.37014

[Ferenczi and Park 2007] S. Ferenczi and K. K. Park, "Entropy dimensions and a class of constructive examples", Discrete Contin. Dyn. Syst. 17:1 (2007), 133-141. MR 2007i:37014 Zbl 1128.37004

[Goodwyn 1972] L. W. Goodwyn, "Some counter-examples in topological entropy", Topology 11 (1972), 377-385. MR 47 \#2575 Zbl 0246.54049

[Hric 1999] R. Hric, "Topological sequence entropy for maps of the interval", Proc. Amer. Math. Soc. 127:7 (1999), 2045-2052. MR 99j:58141 Zbl 0923.26004

[Hric 2000] R. Hric, "Topological sequence entropy for maps of the circle", Comment. Math. Univ. Carolin. 41:1 (2000), 53-59. MR 2001c:37016 Zbl 1039.37007

[Hu 1993] H. Y. Hu, "Some ergodic properties of commuting diffeomorphisms", Ergodic Theory Dynam. Systems 13:1 (1993), 73-100. MR 94b:58061 Zbl 0788.58033

[Huang et al. 2007] W. Huang, K. K. Park, and X. Ye, "Topological disjointness from entropy zero systems”, Bull. Soc. Math. France 135:2 (2007), 259-282. MR 2009g:54084 Zbl 1157.54015

[Huang et al. 2008] X. Huang, X. Wen, and F. Zeng, "Topological pressure of nonautonomous dynamical systems", Nonlinear Dyn. Syst. Theory 8:1 (2008), 43-48. MR 2010a:37027

[Katok and Hasselblatt 1995] A. Katok and B. Hasselblatt, Introduction to the modern theory of dynamical systems, Encyclopedia of Mathematics and its Applications 54, Cambridge University Press, 1995. MR 96c:58055 Zbl 0878.58020 
[Kolyada and Snoha 1996] S. Kolyada and Ł. Snoha, "Topological entropy of nonautonomous dynamical systems", Random Comput. Dynam. 4:2-3 (1996), 205-233. MR 98f:58126 Zbl 0909.54012

[Kolyada et al. 1999] S. Kolyada, M. Misiurewicz, and Ł. Snoha, "Topological entropy of nonautonomous piecewise monotone dynamical systems on the interval", Fund. Math. 160:2 (1999), 161-181. MR 2001g:37017 Zbl 0936.37004

[Kolyada et al. 2004] S. Kolyada, L. Snoha, and S. Trofimchuk, "On minimality of nonautonomous dynamical systems”, Nelīnĭ̄ñ Koliv. 7:1 (2004), 86-92. MR 2006m:37010 Zbl 1092.37011

[Misiurewicz 1981] M. Misiurewicz, "Structure of mappings of an interval with zero entropy", Inst. Hautes Études Sci. Publ. Math. 53 (1981), 5-16. MR 83j:58071 Zbl 0477.58030

[Misiurewicz and Smítal 1988] M. Misiurewicz and J. Smítal, "Smooth chaotic maps with zero topological entropy", Ergodic Theory Dynam. Systems 8:3 (1988), 421-424. MR 90a:58118 Zbl 0689.58028

[Misiurewicz and Szlenk 1980] M. Misiurewicz and W. Szlenk, "Entropy of piecewise monotone mappings”, Studia Math. 67:1 (1980), 45-63. MR 82a:58030 Zbl 0445.54007

[Mouron 2007] C. Mouron, "Positive entropy on nonautonomous interval maps and the topology of the inverse limit space", Topology Appl. 154:4 (2007), 894-907. MR 2008f:37035 Zbl 1117.37011

[Pollicott and Yuri 1998] M. Pollicott and M. Yuri, Dynamical systems and ergodic theory, London Mathematical Society Student Texts 40, Cambridge University Press, 1998. MR 99f:58130 Zbl 0897.28009

[Raith 2004] P. Raith, "Two commuting interval maps with entropy zero whose composition has positive topological entropy", pp. 351-354 in Iteration theory (ECIT '02) (Évora, 2002), edited by J. Sousa Ramos et al., Grazer Mathematische Berichte 346, Karl-Franzens-Univ. Graz, Graz, 2004. MR 2005g:37079 Zbl 1072.37031

[Rothschild 1971] J. G. Rothschild, On the computation of topological entropy, Ph.D. thesis, City University of New York, 1971. MR 2621267

[Walters 1982] P. Walters, An introduction to ergodic theory, Graduate Texts in Mathematics 79, Springer, New York, 1982. MR 84e:28017 Zbl 0475.28009

[Zhu et al. 2006] Y. Zhu, J. Zhang, and L. He, "Topological entropy of a sequence of monotone maps on circles”, J. Korean Math. Soc. 43:2 (2006), 373-382. MR 2007j:37068 Zbl 1098.37038 
Received February 9, 2012. Revised August 12, 2012.

RUI KUANG

DEPARTMENT OF MATHEMATICS

SOUTH CHINA UNIVERSITY OF TECHNOLOGY

510641 GUANGZHOU

CHINA

kuangrui@scut.edu.cn

Wen-Chiao Cheng

DEPARTMENT OF APPLIED MATHEMATICS

Chinese Culture University

YANGMINGSHAN, TAIPEI 11114

TAIWAN

zwq2@faculty.pccu.edu.tw

BING LI

DePartment of Mathematics

SOUTH CHINA UNIVERSITY OF TECHNOLOGY

510641 GUANGZHOU

CHINA

and

Department of MATHEMATICAL SCIENCE

UNIVERSITY OF OULU

P.O. Box 3000

FI-90014 OULU

FINLAND

libing0826@gmail.com 


\title{
PACIFIC JOURNAL OF MATHEMATICS
}

\author{
msp.org/pjm
}

Founded in 1951 by E. F. Beckenbach (1906-1982) and F. Wolf (1904-1989)

\section{EDITORS}

V. S. Varadarajan (Managing Editor)

Department of Mathematics

University of California

Los Angeles, CA 90095-1555

pacific@math.ucla.edu

Paul Balmer

Department of Mathematics

University of California

Los Angeles, CA 90095-1555

balmer@math.ucla.edu

Daryl Cooper

Department of Mathematics

University of California

Santa Barbara, CA 93106-3080 cooper@math.ucsb.edu

Jiang-Hua $\mathrm{Lu}$

Department of Mathematics

The University of Hong Kong

Pokfulam Rd., Hong Kong jhlu@maths.hku.hk
Don Blasius

Department of Mathematics University of California

Los Angeles, CA 90095-1555

blasius@math.ucla.edu

Robert Finn

Department of Mathematics Stanford University

Stanford, CA 94305-2125

finn@math.stanford.edu

Sorin Popa

Department of Mathematics

University of California

Los Angeles, CA 90095-1555

popa@math.ucla.edu

Paul Yang

Department of Mathematics

Princeton University

Princeton NJ 08544-1000

yang@math.princeton.edu

\section{PRODUCTION}

Silvio Levy, Scientific Editor, production@msp.org

\section{SUPPORTING INSTITUTIONS}

ACADEMIA SINICA, TAIPEI

CALIFORNIA INST. OF TECHNOLOGY

INST. DE MATEMÁTICA PURA E APLICADA

KEIO UNIVERSITY

MATH. SCIENCES RESEARCH INSTITUTE

NEW MEXICO STATE UNIV.

OREGON STATE UNIV.

\author{
STANFORD UNIVERSITY \\ UNIV. OF BRITISH COLUMBIA \\ UNIV. OF CALIFORNIA, BERKELEY \\ UNIV. OF CALIFORNIA, DAVIS \\ UNIV. OF CALIFORNIA, LOS ANGELES \\ UNIV. OF CALIFORNIA, RIVERSIDE \\ UNIV. OF CALIFORNIA, SAN DIEGO \\ UNIV. OF CALIF., SANTA BARBARA
}

\author{
Vyjayanthi Chari \\ Department of Mathematics \\ University of California \\ Riverside, CA 92521-0135 \\ chari@math.ucr.edu \\ Kefeng Liu \\ Department of Mathematics \\ University of California \\ Los Angeles, CA 90095-1555 \\ liu@math.ucla.edu \\ Jie Qing \\ Department of Mathematics \\ University of California \\ Santa Cruz, CA 95064 \\ qing@cats.ucsc.edu
}

These supporting institutions contribute to the cost of publication of this Journal, but they are not owners or publishers and have no responsibility for its contents or policies.

See inside back cover or msp.org/pjm for submission instructions.

The subscription price for 2013 is US \$400/year for the electronic version, and \$485/year for print and electronic.

Subscriptions, requests for back issues and changes of subscribers address should be sent to Pacific Journal of Mathematics, P.O. Box 4163, Berkeley, CA 94704-0163, U.S.A. The Pacific Journal of Mathematics is indexed by Mathematical Reviews, Zentralblatt MATH, PASCAL CNRS Index, Referativnyi Zhurnal, Current Mathematical Publications and the Science Citation Index.

The Pacific Journal of Mathematics (ISSN 0030-8730) at the University of California, c/o Department of Mathematics, 798 Evans Hall \#3840, Berkeley, CA 94720-3840, is published monthly except July and August. Periodical rate postage paid at Berkeley, CA 94704, and additional mailing offices. POSTMASTER: send address changes to Pacific Journal of Mathematics, P.O. Box 4163, Berkeley, CA 94704-0163.

PJM peer review and production are managed by EditFLOW ${ }^{\circledR}$ from Mathematical Sciences Publishers.

PUBLISHED BY

mathematical sciences publishers

nonprofit scientific publishing

http://msp.org/

(C) 2013 Mathematical Sciences Publishers 


\section{PACIFIC JOURNAL OF MATHEMATICS}

Volume $262 \quad$ No. $2 \quad$ April 2013

Certifying incompressibility of noninjective surfaces with scl

DANNY CALEGARI

Global well-posedness for the 3D rotating Navier-Stokes equations with highly oscillating initial data

QIONGLEI CHEN, CHANGXING MiAO and ZHIFEI ZHANG

Presenting Schur superalgebras

HOUSSEIN El TURKEY and JONATHAN R. KUJAWA

Classifying zeros of two-sided quaternionic polynomials and computing zeros of two-sided polynomials with complex coefficients

FENG LIANGGUI and ZHAO KAIMING

Coxeter groups, imaginary cones and dominance

XIANG FU

Semicontinuity of automorphism groups of strongly pseudoconvex domains: The low differentiability case

Robert E. Greene, Kang-Tae Kim, Steven G. Krantz and

AERYEONG SEO

Klein four-subgroups of Lie algebra automorphisms

JING-SONG HUANG and JUN YU

Fractal entropy of nonautonomous systems

Rui KuANG, WEN-ChiaO CHENG and BING LI

A GJMS construction for 2-tensors and the second variation of the total

$Q$-curvature

YoshiniKo MaTSUMOTO

Droplet condensation and isoperimetric towers

Matteo Novaga, Andrei Sobolevski and Eugene Stepanov

Brauer's height zero conjecture for metacyclic defect groups

BENJAMIN SAMBALE 